\title{
FIJACIÓN ESPINOPÉLVICA EN EL ADULTO. CRITERIOS DE SELECCIÓN
}

FIXAÇÃO ESPINO-PÉLVICA EM ADULTOS. CRITÉRIOS DE SELEÇÃO

SPINOPELVIC FIXATION IN ADULTS. SELECTION CRITERIA

Tomás Rüdt' ${ }^{1}$ Roberto Gustavo Zisuela², Guillermo KahL ${ }^{2}$, Maximiliano Santi ${ }^{3}$

\section{RESUMEN}

Objetivo: Mencionar nuestros criterios de selección para realizar fijación espinopélvica en patología degenerativa del adulto. Método: Entre 2005 y 2008, analizamos 25 pacientes intervenidos quirúrgicamente con técnica de fijación espinopélvica con tornillos ilíacos. Criterios de selección: 1) Deformidades graves asociadas a desequilibrio espinopélvico. 2) Fusiones multiniveles asociadas a patología degenerativa del primer espacio móvil. 3) Cirugías de revisión: cifoescoliosis o espondilolistesis de alto grado. Se evaluaron edad, sexo, Score de Owestry preoperatorio y posoperatorio y complicaciones. Resultado: La edad fue de 55 a 75 años (promedio 67,8). Dos pacientes eran del (8\%) de sexo masculino y 23 (92\%), del femenino. Promedio de seguimiento: 2,5 años. Score de Owestry, promedio preoperatorio: 67,44\% y posoperatorio: 9,76 \%. Hubo tres complicaciones: infección aguda, pseudoartrosis y aflojamiento de tornillos ilíacos. Conclusión: La fijación espinopélvica con tornillos ilíacos es una técnica sencilla que nos asegura un óptimo anclaje distal para la artrodesis lumbosacra en cirugías de revisión y deformidades complejas.

Descriptores: Vértebras lumbares/cirugía; Fusión vertebral/métodos; Estenosis espinal/cirugía; Espondilolistesis/cirugía; Articulación sacroilíaca.

\begin{abstract}
RESUMO
Objetivo: Mencionar nossos critérios de seleção para realizar fixação espino-pélvica em doença degenerativa da coluna vertebral do adulto. Método: Entre 2005 e 2008 analisamos 25 pacientes submetidos a cirurgia com técnica de fixação espino-pélvica com parafusos ilíacos. Critérios de seleção: 1) Deformidades graves associadas ao desequilíbrio espino-pélvico. 2) Fusões de múltiplos níveis associada a patologia degenerativa do primeiro espaço móvel. 3) Cirurgias de revisão: cifoescoliose ou espondilolistese de alto grau. Avaliou-se idade, sexo, escore de Owestry pré e pós-operatório e complicações. Resultado: A idade foi de 55 a 75 anos (média 67,8 anos). Dois (8\%) pacientes do sexo masculino e 23 (92\%), do sexo feminino. Média de acompanhamento: 2,5 anos. Escore de Owestry pré-operatório médio: 67,44\% e pós-operatório: 9,76\%. Foram constatadas três complicações: infecção aguda, pseudoartrose e afrouxamento dos parafusos ilíacos em um paciente. Conclusão: A fixação espino-pélvica com parafusos ilíacos é uma técnica simples que garante ancoragem distal ideal para a artrodese lombossacral em cirurgias de revisão e deformidades complexas.
\end{abstract}

Descritores: Vértebras lombares/cirurgia; Fusão vertebral/métodos; Estenose espinal/cirurgia; Espondilolistese/cirurgia; Articulação sacroiliaca.

\section{ABSTRACT}

Objective: To point out our selection criteria for spinopelvic fixation in degenerative spine disease in adults. Methods: Between 2005 and 2008,25 patients who underwent lumbosacral spine surgery with spinopelvic fixation with iliac screws were analysed. Selection criteria: 1) Severe deformities associated with pelvic imbalance. 2) Multilevel fusion associated with degenerative disease of the first mobile space. 3) Revision surgeries: Kyphoscoliosis or high- grade spondylolisthesis. We assessed age, sex, pre and postoperative Oswestry Score and complications. Results: Age was 55 to 75 years (mean 67.8). Two patients (8\%) were male and 23 (92\%) female. Mean follow-up: 2.5 years. Preoperative Oswestry Score : 67.44\%; postoperative: 9.76\%. Three complications were found: one acute infection, one pseudoarthrosis, and loosening of iliac screws in one patient. Conclusion: The spinopelvic fixation with iliac screws is a simple technique which allows an optimal distal anchorage in lumbosacral fusions for revision surgeries and complex deformities.

Keywords: Lumbar vertebrae/surgery; Spinal Fusion/methods; Spinal stenosis/surgery; Spondylolisthesis/surgery; Sacroiliac joint.

\section{INTRODUCCIÓN}

La complejidad anatómica, las fuerzas biomecánicas solicitadas y la mala calidad ósea del sacro son algunas de las razones por las cuales la fusión de la charnela lumbosacra continua siendo una tarea desafiante en la cirugía de columna.

El sacro está constituido por cinco vertebras fusionadas y es la llave ósea que une las dos hemipelvis por la articulaciones sacroiliacas. Su diámetro anteroposterior en la mujer va desde aproximadamente $47 \mathrm{~mm}$ en S1 a $28 \mathrm{~mm}$ en S2 y en el hombre de 50 a $31 \mathrm{~mm}$ respectivamente ${ }^{1}$.

La mayor parte del hueso sacro es esponjoso pero hay áreas de mayor densidad como el ala sacra y especialmente el promotorio².

La articulación sacroliliaca contiene una membrana sinovial pero presenta mínima movilidad por sus fuertes estructuras ligamentarias y además por la interdigitación ósea entre el sacro y el hueso iliaco. Su función es la de transmisión de carga axial a cada hemipelvis.

Por la región lumbosacra transcurren numerosas estructuras anatómicas de importancia como la arteria y vena iliaca interna, la arteria y vena sacra media, la cadena simpática, el tronco lumbosacro y el colon sigmoideo. Todas estas estructuras pueden ser lesionadas con la instrumentación lumbopélvica.

El objetivo del presente trabajo es mencionar cuales son los criterios de selección, en nuestro servicio, para realizar fijación espinopélvica en patología degenerativa y cirugías de revisión en deformidades del adulto.

\section{MATERIALES Y MÉTODOS}

Para esta evaluación tomamos, en forma retrospectiva, a 25 pacientes operados por nuestro equipo entre los años 2005 y 2008 que fueron intervenidos quirúrgicamente con técnica de fijación espinopelvica con tornillos iliacos.

En nuestra muestra se tomaron pacientes adultos, de ambos sexos y según la patología a tratar los criterios de exclusión fueron las fracturas, los tumores, las infecciones y las espondilolistesis de alto grado, mientras que los criterios de inclusión fueron las escoliosis y las reoperaciones.

Los criterios de selección para la indicación de fijación espinopélvica en nuestro servicio fueron:

\footnotetext{
1. Jefe de la Unidad Columna Vertebral del Servicio de Traumatología y Ortopédia del Sanatorio Trinidad Mitre, Ciudad Autónona de Buenos Aires, Argentina.

2. Médico Staff de la Unidad Columna del Sanatorio Trinidad Mitre - Buenos Aires, Argentina.
}

3. Médico Fellow de la Unidad Columna del Sanatorio Trinidad Mitre - Buenos Aires, Argentina.

Trabajo realizado en el Sanatorio Trinidad Mitre de Buenos Aires, Unidad de Columna Vertebral del Servicio de Traumatología y Ortopedia.

Correspondência: Sanatorio Trinidad Mitre de Buenos Aires, Unidad de Columna Vertebral del Servicio de Traumatología y Ortopedia. Bartolomé Mitre 2553, C.A.B.A., Argentina. Email: tomasrudt@hotmail.com - guillermokahl@yahoo.com.ar 
Deformidades severas asociadas a desbalance espinopélvico. Fusiones multinivel asociada a patología degenerativa del primer espacio móvil.

Cirugías de revisión en deformidades: cifoescoliosis o espondilolistesis de alto grado.

Los estudios complementarios imagenológicos preoperatorios solicitados fueron: Espinograma frente y perfil, RX oblicuas y dinámicas (flexoextensión y bendings laterales), R.M.N. y T.A.C. de columna.

El seguimiento postoperatorio se realizó clínicamente, con radiografías postoperatorias inmediatas, espinogramas seriados al mes, tres meses, seis meses y al año y T.A.C. a los seis meses.

En todos los casos se evaluó la edad, la distribución por sexo, la evaluación clínica preoperatoria y postoperatoria según el Score de Owestry y las complicaciones.

\section{RESULTADOS}

La edad de los pacientes osciló entre los 55 y los 75 años con un promedio de 67.8 años. Dos (8\%) pacientes fueron de sexo masculino y 23 (92\%) de sexo femenino.

El promedio de seguimiento postoperatorio fue de 2.5 años con una rango de 1.5 a 3 años.

Según la evaluación de los pacientes con el Score de Owestry el preoperatorio promedio fue del $67.44 \%$ mientras que el postoperatorio promedio fue del $9.76 \%$. Revelando una franca mejoría clínica de los pacientes tratados quirúrgicamente (Tabla 1).

Hubo tres complicaciones encontradas: una infección aguda que fue tratada con limpieza quirúrgica y antibióticoterapia prolongada, una pseudoartrosis sintomática asociada a rotura del implante donde se realizó una reoperación con recambio de barras y tornillos, cruentado del lecho y colocación de injerto óseo autólogo y por último un aflojamiento de tornillos iliacos asintomático donde no se tomó ninguna conducta.

\section{DISCUSIÓN}

McCord et al. ${ }^{3}$ desde la biomecánica del raquis, definió el punto de Pivot Lumbosacro como el punto medio de la columna osteoligamentaria entre la última vertebra lumbar y el sacro. En su trabajo fueron comparados distintos tipos de implantes con fijación S1, S2 e iliaco observando mayor resistencia del mismo con tornillos iliacos. Por ende concluye que cuanto más se extiende el implante anterior a el punto de Pivot Lumbosacro más fuerte será su construcción.

O 'Brien ${ }^{4}$ dividió a la región sacropélvica en tres zonas siendo la zona 1 la que abarca el cuerpo vertebral de S1 y el margen cefálico del ala sacra, la zona 2 comprende el resto del hueso sacro y el coxis y finalmente la zona 3 corresponde a ambos huesos iliacos.

Además observó que la fijación del implante va mejorando progresivamente de la zona 1 a la zona 3 . Siendo, desde el punto de vista biomecánico, esta última zona la que ofrece mayor resistencia del implante a las fuerzas de pull-out y a los movimientos de bending en la charnela lumbosacra.

Existen publicaciones que concluyen en que las instrumentaciones pediculares y alares al sacro tienen poco control de la flexión en la charnela lumbosacra ${ }^{5}$ originando complicaciones como falla del implante de hasta el $70 \%$, pull-out de tornillos pediculares sacros

\section{REFERENCIAS}

1. Asher MA, Strippgen WE. Anthropometric studies of the human sacrum relating to dorsal transsacral implant designs. Clin Orthop Relat Res. 1986;(203):58-62.

2. Peretz AM, Hipp JA, Heggeness MH. The internal bony architecture of the sacrum. Spine (Phila Pa 1976). 1998:23(9):971-4.

3. McCord DH, Cunningham BW, ShonoY, Myers JJ, McAfee PC. Biomechanical analysis of lumbosacral fixation. Spine (Phila Pa 1976). 1992;17(Suppl 8):S235-43.

4. O'Brien MF. Sacropelvic fixation in spinal deformity. In: De Wald RL, editor. Spinal deformities: the comprehensive text. New York: Thieme; 2003. p. 601-14.

5. Camp JF, Caudle R, Ashmun RD, Roach J. Immediate complications of Cotrel-Dubousset instrumentation to the sacro-pelvis. A clinical and biomechanical study. Spine (Phila Pa 1976). 1990;15(9):932-41 6. Devlin VJ, Boachie-Adjei O, Bradford DS, Ogilvie JW, Transfeldt EE. Treatment of adult spinal deformity with fusion to the sacrum using CD instrumentation. J Spinal Disord. 1991:4(1):1-14

7. Emami A, Deviren V, Berven S, Smith JA, Hu SS, Bradford DS. Outcome and complications of long fusions to the sacrum in adult spine deformity: luque-galveston, combined iliac and sacral screws, and sacral fixation. Spine (Phila Pa 1976). 2002 1;27(7):776-86.

8. Kim YJ, Bridwell KH, Lenke LG, Cho KJ, Edwards CC 2nd, Rinella AS. Pseudarthrosis in adult spinal deformity following multisegmental instrumentation and arthrodesis. J Bone Joint Surg Am.2006;88(4:721-8.

9. Allen $\mathrm{BL} J \mathrm{Jr}$, Ferguson $\mathrm{RL}$. The Galveston technique of pelvic fixation with L-rod instrumentation of the spine. Spine (Phila Pa 1976). 1984;9(4):388-94

10. CotrelY, Dubousset J. New segmental posterior instrumentation of the spine. [Abstract]. Orthop Trans. 1985;9:118.
Tabla 1. Pacientes, edad, sexo, Score de Owestry, patología y complicaciones.

\begin{tabular}{c|c|c|c|c|c|c}
\hline Pte & Edad & Sexo & Score de Owestry & Score de Owestry & Patología & Complicaciones \\
\hline & & & Preop & Postop & & \\
\hline 1 & 65 & Fem & $48 \%$ & $4 \%$ & Escoliosis & \\
\hline 2 & 62 & Fem & $60 \%$ & $4 \%$ & Escoliosis & \\
\hline 3 & 72 & Fem & $78 \%$ & $20 \%$ & Reoperación & Infección \\
\hline 4 & 75 & Fem & $84 \%$ & $24 \%$ & Cifoescoliosis & Pseudoartrosis \\
\hline 5 & 68 & Fem & $52 \%$ & $12 \%$ & Escoliosis & \\
\hline 6 & 70 & Fem & $78 \%$ & $18 \%$ & Cifoescoliosis & \\
\hline 7 & 67 & Fem & $60 \%$ & $4 \%$ & Escoliosis & \\
\hline 8 & 71 & Fem & $58 \%$ & $8 \%$ & Escoliosis & \\
\hline 9 & 58 & Fem & $70 \%$ & $12 \%$ & Reoperación & \\
\hline 10 & 73 & Fem & $82 \%$ & $24 \%$ & Escoliosis & Aflojamiemto \\
\hline 11 & 71 & Fem & $62 \%$ & $8 \%$ & Reoperación & \\
\hline 12 & 55 & Mas & $78 \%$ & $20 \%$ & Cifoescoliosis & \\
\hline 13 & 66 & Fem & $68 \%$ & $2 \%$ & Escoliosis & \\
\hline 14 & 74 & Fem & $82 \%$ & $8 \%$ & Cifoescoliosis & \\
\hline 15 & 59 & Fem & $74 \%$ & $10 \%$ & Escoliosis & \\
\hline 16 & 72 & Fem & $60 \%$ & $8 \%$ & Escoliosis & \\
\hline 17 & 69 & Fem & $76 \%$ & $10 \%$ & Escoliosis & \\
\hline 18 & 71 & Fem & $68 \%$ & $8 \%$ & Escoliosis & \\
\hline 19 & 72 & Mas & $70 \%$ & $10 \%$ & Cifoescoliosis & \\
\hline 20 & 68 & Fem & $52 \%$ & $4 \%$ & Escoliosis & \\
\hline 21 & 66 & Fem & $62 \%$ & $2 \%$ & Escoliosis & \\
\hline 22 & 70 & Fem & $58 \%$ & $4 \%$ & Escoliosis & \\
\hline 23 & 65 & Fem & $70 \%$ & $8 \%$ & Escoliosis & \\
\hline 24 & 67 & Fem & $74 \%$ & $14 \%$ & Cifoescoliosis & \\
\hline 25 & 69 & Fem & $62 \%$ & $2 \%$ & Escoliosis & \\
\hline
\end{tabular}

de hasta el $44 \%$ y pseudoartrosis de hasta el $33 \%$ 6-8

Muchas de las complicaciones mencionadas fueron disminuidas con la técnica de Glaveston desarrollada por Allen y Ferguson ${ }^{9}$ en la época de los ochenta. Eran utilizadas barras contorneadas insertadas desde la espina iliaca posterosuperior, dentro de cada hueso pélvico, orientadas hacia la región por encima de la escotadura ciática. Las partes proximales de las barras primero fueron sujetas a la columna con alambres sublaminares como en la técnica de Luque y luego con tornillos pediculares y ganchos según los principios de la instrumentación de Cotrel-Dubousset ${ }^{10-12}$

Luego fue desarrollada la instrumentación con tornillos ilia$\cos ^{13,14}$. Este sistema tiene la ventaja de ser de fácil colocación, se pueden colocar más de un tornillo por lado y tiene mejor interdigitación en el hueso iliaco que las barras de Galveston con el consiguiente menor índice de pull-out ${ }^{15-17}$. Además en un trabajo cadavérico se comprobó que los tornillos iliacos eran más de tres veces más fuerte que las barras transiliacas de Galveston ${ }^{18,19}$

\section{CONCLUSIONES}

La fijación espinopélvica con tornillos iliacos es una técnica sencilla que nos asegura un óptimo anclaje distal para la artrodesis lumbosacra en cirugías de revisión y deformidades complejas.

En nuestra experiencia siguiendo los criterios de selección mencionados para la indicación de fijación espinopélvica consideramos que es una técnica con resultados clínicos e imagenológicos satisfactorios que indudablemente mejoran la calidad de vida del paciente.

11. Camp JF, Caudle R, Ashmun RD, Roach J. Immediate complications of Cotrel-Dubousset instrumentation to the sacro-pelvis. A clinical and biomechanical study. Spine (Phila Pa 1976). 1990;15(9):932-41

12. Winter RB, Denis F, Lonstein JE, Dezen E. Salvage and reconstructive surgery for spinal deformity using Cotrel-Dubousset instrumentation. Spine (Phila Pa 1976). 1991·16(Suppl 8):S412-7.

13. O'Brien JR, YuWD, Bhatnagar R, Sponseller P, Kebaish KM. An anatomic study of the S2 iliac technique for lumbopelvic screw placement. Spine (Phila Pa 1976).2009 20;34(12):E439-42

14. SchildhauerTA, McCulloch P, Chapman JR, Mann FA. Anatomic and radiographic considerations for placement of transiliac screws in lumbopelvic fixations. J Spinal Disord Tech. 2002;15(3):199-205.

15. Kuklo TR, Bridwell KH, Lewis SJ, Baldus C, Blanke K, Iffrig TM, et al. Minimum 2-year analysis of sacropelvic fixation and L5-S1 fusion using S1 and iliac screws. Spine (Phila Pa 1976). 2001;26(18):1976-83.

16. Peelle MW, Lenke LG, Bridwell KH, Sides B. Comparison of pelvic fixation techniques in neuromuscular spinal deformity correction: Galveston rod versus iliac and lumbosacral screws. Spine (Phila Pa 1976). 2006 15;31(20):2392-8;

17. Tsuchiya K, Bridwell KH, KukloTR, Lenke LG, Baldus C. Minimum 5-year analysis of L5-S1 fusion using sacropelvic fixation (bilateral S1 and iliac screws) for spinal deformity. Spine (Phila Pa 1976). 2006 1;31(3):303-8.

18. Balderston RA, Winter RB, Moe JH, Bradford DS, Lonstein JE. Fusion to the sacrum for nonparalytic scoliosis in the adult. Spine (Phila Pa 1976). 1986;11(8):824-9.

19. Schwend RM, Sluyters R, Najdzionek J. The pylon concept of pelvic anchorage for spinal instrumentation in the human cadaver. Spine (Phila Pa 1976). 2003 15;28(6):542-7. 